\title{
RESIDUALLY FINITE NON-EXACT GROUPS
}

\author{
DAMIAN OSAJDA
}

\begin{abstract}
We construct the first examples of residually finite non-exact groups.
\end{abstract}
\section{Introduction}

A finitely generated group is non-exact if its reduced $C^{*}$-algebra is non-exact. Equivalently, it has no Guoliang Yu's property A (see e.g. [Roe03, Chapter 11.5]). Most classical groups are exact, that is, are not non-exact. The first examples of nonexact groups were the so-called Gromov monsters [Gro03]. In this paper we rely on author's construction of groups containing isometrically expanders [Osa14]. The isometric embedding of an expanding family of graphs performed in the latter construction is possible thanks to using a graphical small cancellation. That particular construction is crucial for results in the current paper.

Main Theorem. There exist finitely generated residually finite non-exact groups defined by infinite graphical small cancellation presentations.

This answers one of few questions from the Open Problems chapter of the BrownOzawa book [BO08, Problem 10.4.6]. Some motivations for the question can be found there. Our interest in the problem is twofold: First, we plan to use residually finite non-exact groups constructed here for producing other, essentially new examples of non-exact groups; Second, we believe that our examples might be useful for constructing and studying metric spaces with interesting new coarse geometric features. More precisely, let $G$ be a finitely generated infinite residually finite group, and let $\left(N_{i}\right)_{i=1}^{\infty}$ be a sequence of its finite index normal subgroups with $\bigcap_{i=1}^{\infty} N_{i}=\{1\}$. The box space of $G$ corresponding to $\left(N_{i}\right)$ is the coarse disjoint union $\bigsqcup_{i=1}^{\infty} G / N_{i}$, with each $G / N_{i}$ endowed with the word metric coming from a given finite generating set for $G$. Properties of the group $G$ are often related to coarse geometric properties of its box space. For example, a group is amenable iff its box space has property A [Roe03, Proposition 11.39]. Box spaces provide a powerful method for producing metric spaces with interesting coarse geometric features (see e.g. [Roe03, Chapter

Keywords and phrases: Group exactness, Residual finiteness, Graphical small cancellation Mathematics Subject Classification: 20F69, 20F06, 46B85 
11.3]). The groups constructed in the current article open a way to studying box spaces of non-exact groups and make the following questions meaningful.

Questions. What are coarse geometric properties of box spaces of non-exact groups? Can non-exactness of a group be characterized by coarse geometric properties of its box space? 1

The idea of the construction of groups as in the Main Theorem is as follows. The group is defined by an infinite graphical small cancellation presentation. It is a limit of a direct sequence of groups $G_{i}$ with surjective bonding maps - each $G_{i}$ has a graphical small cancellation presentation being a finite chunk of the infinite presentation. Such finite chunks are constructed inductively, using results of [Osa14], so that they satisfy the following conditions. Each group $G_{i}$ is hyperbolic and acts geometrically on a CAT(0) cubical complex, hence it is residually finite. ${ }^{2}$ For every $i$, there exists a map $\varphi_{i}: G_{i} \rightarrow F_{i}$ to a finite group such that no nontrivial element of the $i$-ball around identity is mapped to 1 . Every $\varphi_{i}$ factors through the quotient maps $G_{i} \rightarrow G_{j}$ so that it induces a map of the limit group $G$ to a finite group injective on a large ball. The residual finiteness of $G$ follows. Finally, $G$ is non-exact since its Cayley graph contains a sequence of graphs (relators) without property A.

In Sect. 2 we present preliminaries on graphical small cancellation presentations and we recall some results from [Osa14]. In Sect. 3 we present the inductive construction of the infinite graphical small cancellation presentation proving the Main Theorem.

\section{Preliminaries}

We follow closely (up to the notation) [Osa14].

2.1 Graphs. All graphs considered in this paper are simplicial, that is, they are undirected, have no loops nor multiple edges. In particular, we will consider Cayley graphs of groups, denoted $\operatorname{Cay}(G, S)$ - the Cayley graph of $G$ with respect to the generating set $S$. For a set $S$, an $S$-labelling of a graph $\Theta$ is the assignment of elements of $S \cup S^{-1}\left(S^{-1}\right.$ being the set of formal inverses of elements of $S$ ) to directed edges (pairs of vertices) of $\Theta$, satisfying the following condition: If $s$ is assigned to $(v, w)$ then $s^{-1}$ is assigned to $(w, v)$. All labellings considered in this paper are reduced: If $s$ is assigned to $(v, w)$, and $s^{\prime}$ is assigned to $\left(v, w^{\prime}\right)$ then $s=s^{\prime}$ iff $w=w^{\prime}$. For a covering of graphs $p: \widehat{\Theta} \rightarrow \Theta$, having a labelling $(\Theta, l)$ we will always consider the induced labelling $(\widehat{\Theta}, \widehat{l})$ : the label of an edge $e$ in $\widehat{\Theta}$ is the same

\footnotetext{
1 After circulating the first version of the article I was informed that Thibault Pillon introduced a notion of "fibred property A", and proved that a finitely generated residually finite group is exact iff its box space has this property (unpublished).

${ }^{2}$ Note that Pride [Pri89] constructed infinitely presented classical small cancellation groups that are not residually finite. They are limits of hyperbolic CAT(0) cubical (hence residually finite) groups.
} 
as the label of $p(e)$. Speaking about the metric on a connected graph $\Theta$ we mean the metric space $\left(\Theta^{(0)}, d\right)$, where $\Theta^{(0)}$ is the set of vertices of $\Theta$ and $d$ is the path metric. The ball of radius $i$ around $v$ in $\Theta$ is $B_{i}(v, \Theta):=\left\{w \in \Theta^{(0)} \mid d(w, v) \leqslant i\right\}$. In particular, the metric on $\operatorname{Cay}(G, S)$ coincides with the word metric on $G$ given by $S$.

2.2 Graphical small cancellation. A graphical presentation is the following data: $\mathcal{P}=\langle S \mid(\Theta, l)\rangle$, where $S$ is a finite set - the generating set, and $(\Theta, l)$ is a graph $\Theta$ with an $S$-labelling $l$. We assume that $\Theta$ is a disjoint (possibly infinite) union of finite connected graphs with no separating edges $\left(\Theta_{i}\right)_{i \in I}$, and the labelling $l$ restricted to $\Theta_{i}$ is denoted by $l_{i}$. We write $(\Theta, l)=\left(\Theta_{i}, l_{i}\right)_{i \in I}$. A graphical presentation $\mathcal{P}$ defines a group $G:=F(S) / R$, where $R$ is the normal closure in $F(S)$ of the subgroup generated by words in $S \cup S^{-1}$ read along (directed) loops in $\Theta$.

A piece is a labelled path occurring in two distinct connected components $\Theta_{i}$ and $\Theta_{j}$, or occurring in a single $\Theta_{i}$ in two places not differing by an automorphism of $\left(\Theta_{i}, l_{i}\right)$. Observe that if $\left(\widehat{\Theta}_{i}, \widehat{l}_{i}\right) \rightarrow\left(\Theta_{i}, l_{i}\right)$ is a normal covering then two lifts of a path in $\Theta_{i}$ differ by a covering automorphism. In particular, if the covering corresponds to a characteristic subgroup of $\pi_{1}\left(\Theta_{i}\right)$ then a lift of a non-piece is a non-piece.

For $\lambda \in(0,1 / 6]$, the labelling $(\Theta, l)$ or the presentation $\mathcal{P}$ are called $C^{\prime}(\lambda)$-small cancellation if length of every piece appearing in $\Theta_{i}$ is strictly less than $\lambda \operatorname{girth}\left(\Theta_{i}\right)$, where $\operatorname{girth}\left(\Theta_{i}\right)$ is the length of a shortest simple cycle in $\Theta_{i}$. Such presentations define infinite groups. The introduction of graphical small cancellation is attributed to Gromov [Gro03]. For more details see e.g. [Wis17, Osa14]. We will use mostly results proven already in [Wis17, Osa14], so we list only the most important features of groups defined by graphical small cancellation presentations.

First, observe that if $(\Theta, l)$ is a $C^{\prime}(\lambda)$-small cancellation labelling, and $\widehat{\Theta}_{i} \rightarrow \Theta_{i}$ is a covering corresponding to a characteristic subgroup of $\pi_{1}\left(\Theta_{i}\right)$, for each $i$, then the induced labelling $(\widehat{\Theta}, \widehat{l})$ is also $C^{\prime}(\lambda)$-small cancellation. The following result was first stated by Gromov.

Lemma 2.1. ([Gro03]) Let $G$ be the group defined by a graphical $C^{\prime}(\lambda)$-small cancellation presentation $\mathcal{P}$, for $\lambda \in(0,1 / 6]$. Then, for every $i$, there is an isometric embedding $\Theta_{i} \rightarrow \operatorname{Cay}(G, S)$.

The isometric embedding above is just an embedding of $S$-labelled graphs.

2.3 Walls in graphs. A wall in a connected graph is a collection of edges such that removing all interiors of these edges decomposes the graph in exactly two connected components. There are many ways for defining walls in finite graphs $\Theta_{i}$. We would like however that such walls "extend" to walls in $\operatorname{Cay}(G, S)$. We use a particular construction of walls in finite graphs. For such graph $\Theta_{i}$, let $\widehat{\Theta}_{i}$ denote its $\mathbb{Z}_{2}$-homology cover, that is, a normal cover corresponding to the kernel of the obvious map $\pi_{1}\left(\Theta_{i}, v_{0}\right) \rightarrow H_{1}\left(\Theta_{i} ; \mathbb{Z}_{2}\right)$. Wise [Wis17] observed that there is a natural structure of walls on $\widehat{\Theta}_{i}$ : for every edge of $\Theta_{i}$ its preimage is a wall in $\widehat{\Theta}_{i}$. We call these walls $\mathbb{Z}_{2}$-homology cover walls. We will use results of Wise [Wis17] to show 
that such walls, defined for $\widehat{\Theta}_{1}, \ldots, \widehat{\Theta}_{i}$ give rise to walls in $\operatorname{Cay}(\widehat{G}, S)$, where $\widehat{G}$ is the group with the graphical presentation $\left\langle S \mid\left(\widehat{\Theta}_{1}, \widehat{l}_{1}\right), \ldots,\left(\widehat{\Theta}_{i}, \widehat{l}_{i}\right)\right\rangle$, for $\widehat{l}_{j}$ being the labeling induced from $l_{j}$. Furthermore, we show that $\widehat{G}$ acts geometrically on the associated $\mathrm{CAT}(0)$ cube complex. We begin with a technical lemma. All the walls here are $\mathbb{Z}_{2}$-homology cover walls.

LEMMA 2.2. Let $\widehat{\gamma}$ be a geodesic in $\widehat{\Theta}_{i}$ whose first and last edges, $\widehat{e}_{1}$ and $\widehat{e}_{2}$, respectively, belong to walls $w_{1}$ and $w_{2}$. Suppose there is a wall $w$ such that one of its edges $\widehat{e}$ belongs to $\widehat{\gamma}$, and another edge $\widehat{e}^{\prime}$ is (girth $\left.\left(\Theta_{i}\right) / 24\right)$-close to $\widehat{e}_{2}$. Then there is a wall $w^{\prime}$ separating $\widehat{e}_{1}$ and $\widehat{e}_{2}$ but such that no edge of $w^{\prime}$ is (girth $\left.\left(\Theta_{i}\right) / 24\right)$-close to an edge of $w_{1}$ or $w_{2}$.

Proof. By definition $w_{1}, w_{2}$, and $w$ consist of preimages of edges $e_{1}, e_{2}$, and $e$ in $\Theta_{i}$, respectively. Since $\widehat{e}$ and $\widehat{e}^{\prime}$ are in the same wall, but distinct, it follows that their distance is at least girth $\left(\Theta_{i}\right)-1$. Hence the length of $\widehat{\gamma}$ is at least (girth $\left(\Theta_{i}\right)-1-$ girth $\left.\left(\Theta_{i}\right) / 24\right)>\operatorname{girth}\left(\Theta_{i}\right) / 2$. Consider a projection $\gamma$ of $\widehat{\gamma}$. It is a path (sequence of edges) of length (number of edges) at least girth $\left(\Theta_{i}\right) / 2$, without back-tracks. Hence, there exists an edge $f$ in $\gamma$ that is not (girth $\left(\Theta_{i}\right) / 24$ )-close to $e_{1}$ or $e_{2}$ and that is passed by $\gamma$ an odd number of times. The wall $w^{\prime}$ defined by $f$, that is, the wall consisting of preimages of $f$ is the desired wall separating $\widehat{e}_{1}$ and $\widehat{e}_{2}$.

Remark. The above lemma is needed to use [Wis17, Theorem 5.40 and Remark $5.41]^{3}$ in the sequel. Wise's work concerns the so-called cubical small cancellation. It is a far-going generalization of the graphical small cancellation used in the current article (see e.g. [Wis17, 3.s. Examples on p. 72 or Section 5.k. p. 124]). For example, in our case Wise's hyperplanes reduce just to edges, and there exist only cone pieces. Consequently, many assumptions appearing in formulations of results in [Wis17] are easily satisfied in the graphical small cancellation case.

Let $\lambda \in(0,1 / 24]$, and let $\left(\Theta_{1}, l_{1}\right), \ldots,\left(\Theta_{i}, l_{i}\right)$ be a $C^{\prime}(\lambda)$-small cancellation labelling. Then we call the system of $\mathbb{Z}_{2}$-homology walls on $\left(\widehat{\Theta}_{1}, \widehat{l}_{1}\right), \ldots,\left(\widehat{\Theta}_{i}, \widehat{l}_{i}\right)$ a proper $\mathbb{Z}_{2}$-walling. The following lemma is our main tool for proving residual finiteness of intermediate steps in our construction.

LEMMA 2.3. Let $\left(\widehat{\Theta}_{1}, \widehat{l}_{1}\right), \ldots,\left(\widehat{\Theta}_{i}, \widehat{l}_{i}\right)$ be equipped with a proper $\mathbb{Z}_{2}$-walling. Then the group $\widehat{G}=\left\langle S \mid\left(\widehat{\Theta}_{1}, \widehat{l}_{1}\right), \ldots,\left(\widehat{\Theta}_{i}, \widehat{l}_{i}\right)\right\rangle$ acts geometrically on a CAT(0) cubical complex.

Proof. We use [Wis17, Theorem 5.40 and Remark 5.41] for proving that $\widehat{G}$ acts properly on a $\mathrm{CAT}(0)$ cubical complex. We verify that the graphical presentation $\mathcal{P}:=\left\langle S \mid\left(\widehat{\Theta}_{1}, \widehat{l}_{1}\right), \ldots,\left(\widehat{\Theta}_{i}, \widehat{l}_{i}\right)\right\rangle$ fulfills the conditions (1), (2), and (3) from Theorem 5.40 there.

Condition (1) of [Wis17, Theorem 5.40]. We have to show that $\mathcal{P}$ satisfies the generalized $B(6)$ condition of [Wis17, Definition 5.1] and has short innerpaths.

3 Here and everywhere we refer to the preprint version of [Wis17] dated June 19, 2017. 
[Wis17, Lemma 3.67] shows that $\mathcal{P}$ has short innerpaths.

We now turn to [Wis17, Definition 5.1]. Points (1), (2), and (5) in this definition are obvious. For (3) and (4) observe that pieces in $\widehat{\Theta}_{j}$ have length at most (girth $\left.\left(\Theta_{j}\right) / 24\right)$ and distinct edges in the same wall in $\widehat{\Theta}_{j}$ are at distance at least (girth $\left.\left(\Theta_{j}\right)-1\right)$ (compare the proof of Lemma 2.2 above). Hence, (3) and (4) follow as in [Wis17, Remark 5.2].

Condition (2) of [Wis17, Theorem 5.40]. Observe that pieces in each $\widehat{\Theta}_{j}$ have length at most (girth $\left.\left(\Theta_{j}\right) / 24\right)$. Hence the condition follows immediately from Lemma 2.2.

Condition (3) of [Wis17, Theorem 5.40]. This condition is trivially satisfied since every $\widehat{\Theta}_{j}$ is finite.

Therefore, by [Wis17, Theorem 5.40 and Remark 5.41] we conclude that $\widehat{G}$ acts metrically properly on the associated $\mathrm{CAT}(0)$ cubical complex.

Since $\widehat{G}$ is hyperbolic, the cocompactness follows e.g. from [HW14, Lemma 7.2].

\section{The construction}

Fix $\lambda \in(0,1 / 24]$ and a natural number $D \geqslant 3$. Let $(\Theta, l)=\left(\Theta_{i}, l_{i}\right)_{i=1}^{\infty}$ be a sequence of $D$-regular graphs with a labelling $l$ satisfying the following stronger version of $C^{\prime}(\lambda)$-small cancellation: every path in $\Theta_{i}$ of length greater or equal to $\lambda \operatorname{girth}\left(\Theta_{i}\right)$ has labelling different from any other path. Such sequences are constructed in [Osa14].

We will construct a sequence $(\widehat{\Theta}, \widehat{l})=\left(\widehat{\Theta}_{i}, \widehat{l}_{i}\right)_{i=1}^{\infty}$ of normal covers of $\left(\Theta_{i}, l_{i}\right)_{i=1}^{\infty}$ with the induced labelling $\widehat{l}$. By $G_{i}$ we will denote the finitely presented group given by the graphical presentation $G_{i}=\left\langle S \mid \widehat{\Theta}_{1}, \widehat{\Theta}_{2}, \ldots, \widehat{\Theta}_{i}\right\rangle$. The associated quotient maps will be $q_{i}^{j}: G_{i} \rightarrow G_{j}$, with $q_{i}^{i+1}$ denoted $q_{i}$. At the same time we will construct maps to finite groups $\varphi_{i}^{j}: G_{i} \rightarrow F_{j}$, for $i \geqslant j$ with $\varphi_{i}^{i}$ denoted $\varphi_{i}$. We will denote $G=\underline{\lim }\left(G_{i}, q_{i}^{j}\right)$, with $q_{i}^{\infty}: G_{i} \rightarrow G$, and $\varphi_{\infty}^{i}: G \rightarrow F_{i}$ being the induced maps.

We require that the labelled graphs $\left(\widehat{\Theta}_{i}, \widehat{l}_{i}\right)_{i=1}^{\infty}$, and the maps $\varphi_{i}^{j}: G_{i} \rightarrow F_{j}$ satisfy the following conditions:

(A) $\left(\widehat{\Theta}_{1}, \widehat{l}_{1}\right),\left(\widehat{\Theta}_{2}, \widehat{l}_{2}\right), \ldots$ is a $C^{\prime}(\lambda)$-small cancellation labelling;

(B) $\left(\widehat{\Theta}_{1}, \widehat{l}_{1}\right),\left(\widehat{\Theta}_{2}, \widehat{l}_{2}\right), \ldots,\left(\widehat{\Theta}_{i}, \widehat{l}_{i}\right)$ admits a proper $\mathbb{Z}_{2}$-walling for every $i$;

$(C) \varphi_{j}(g) \neq 1$, for every $j$ and every $g \in B_{j}\left(1, \operatorname{Cay}\left(G_{j}, S\right)\right) \backslash\{1\}$;

(D) $\varphi_{l}^{j} \circ q_{k}^{l}=\varphi_{k}^{j}$, for all $j \leqslant k \leqslant l$. 
In particular, the diagram below is commutative.

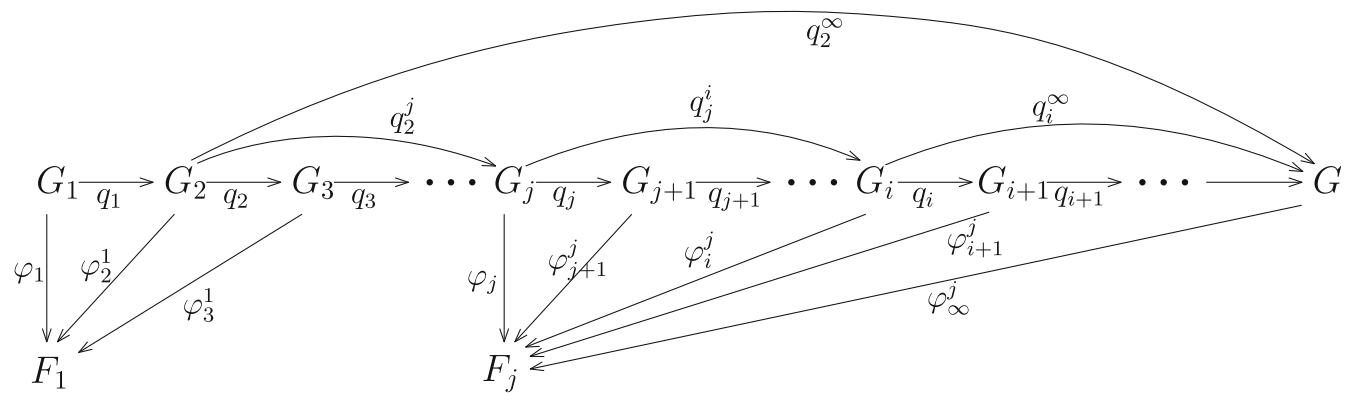

We construct the graphs $\widehat{\Theta}_{i}$, the finite groups $F_{i}$, and the maps $\varphi_{i}^{j}(j \leqslant i)$ inductively, with respect to $i$.

3.1 Induction basis. Let $\widehat{\Theta}_{1}$ be the $\mathbb{Z}_{2}$-homology cover of $\Theta_{1}$ (such a cover corresponds to a characteristic subgroup of $\left.\pi_{1}\left(\Theta_{1}\right)\right)$. Then the following conditions are satisfied:

$\left(A_{1}\right)\left(\widehat{\Theta}_{1}, \widehat{l}_{1}\right),\left(\Theta_{2}, l_{2}\right),\left(\Theta_{3}, l_{3}\right), \ldots$ is a $C^{\prime}(\lambda)$-small cancellation labelling;

$\left(B_{1}\right)\left(\widehat{\Theta}_{1}, \widehat{l}_{1}\right)$ admits a proper $\mathbb{Z}_{2}$-walling.

Then $G_{1}:=\left\langle S \mid \widehat{\Theta}_{1}\right\rangle$ is hyperbolic and, by Lemma 2.3, acts geometrically on a CAT(0) cubical complex. Therefore, by results of Wise [Wis17] and Agol [Ago13] it is residually finite. Let $\varphi_{1}: G_{1} \rightarrow F_{1}$ be a map into a finite group $F_{1}$ such that:

$\left(C_{1}\right) \varphi_{1}(g) \neq 1$ for all $g \in B_{1}\left(1, \operatorname{Cay}\left(G_{1}, S\right)\right) \backslash\{1\}$.

3.2 Inductive step. Assume that the graphs $\widehat{\Theta}_{1}, \widehat{\Theta}_{2}, \ldots, \widehat{\Theta}_{i}$, the finite groups $F_{1}, F_{2}, \ldots, F_{i}$, and the maps $\varphi_{j}^{k}: G_{j} \rightarrow F_{k}$, for $k \leqslant j \leqslant i$ with the following properties have been constructed.

$\left(A_{i}\right)\left(\widehat{\Theta}_{1}, \widehat{l}_{1}\right), \ldots,\left(\widehat{\Theta}_{i}, \widehat{l}_{i}\right),\left(\Theta_{i+1}, l_{i+1}\right),\left(\Theta_{i+2}, l_{i+2}\right), \ldots$ is a $C^{\prime}(\lambda)$-small cancellation labelling;

$\left(B_{i}\right)\left(\widehat{\Theta}_{1}, \widehat{l}_{1}\right), \ldots,\left(\widehat{\Theta}_{i}, \widehat{l}_{i}\right)$ admits a proper $\mathbb{Z}_{2}$-walling;

$\left(C_{i}\right) \varphi_{j}(g) \neq 1$, for every $j \leqslant i$ and every $g \in B_{j}\left(1, \operatorname{Cay}\left(G_{j}, S\right)\right) \backslash\{1\}$;

$\left(D_{i}\right) \varphi_{l}^{j} \circ q_{k}^{l}=\varphi_{k}^{j}$, for all $j \leqslant k \leqslant l \leqslant i$ (that is, the part of the above diagram with all indexes at most $i$ is commutative).

Note that the condition $\left(D_{1}\right)$ is satisfied trivially.

Let $H_{i}$ be a subgroup of $G_{i}$ generated by (the images by $F(S) \rightarrow G_{i}$ of) all the words read along cycles in $\left(\Theta_{i+1}, l_{i+1}\right)$. The subgroup $K_{i}:=\bigcap_{j \leqslant i} \operatorname{ker}\left(\varphi_{i}^{j}\right) \triangleleft G_{i}$ is of finite index. Therefore $H_{i} \cap K_{i}<H_{i}$ is of finite index and we can find a finite normal cover $\bar{\Theta}_{i+1}$ of $\Theta_{i+1}$ such that the normal closure in $G_{i}$ of the subgroup generated by words read along $\left(\bar{\Theta}_{i+1}, \bar{l}_{i+1}\right)$ is contained in $K_{i}$, where $\bar{l}_{i+1}$ is the labelling of $\bar{\Theta}_{i+1}$ induced by $l_{i+1}$ via the covering map. Labelled paths of length greater or equal $\lambda \operatorname{girth}\left(\bar{\Theta}_{i+1}\right)$ in $\left(\bar{\Theta}_{i+1}, \bar{l}_{i+1}\right)$ do not appear in $\left(\widehat{\Theta}_{j}, \widehat{l}_{j}\right)$ for $j \leqslant i$, neither in $\left(\Theta_{j}, l_{j}\right)$ 
for $j \geqslant i+2$. Any two such paths in $\left(\bar{\Theta}_{i+1}, \bar{l}_{i+1}\right)$ differ by a covering automorphism. Therefore, $\left(\widehat{\Theta}_{1}, \widehat{l}_{1}\right), \ldots,\left(\widehat{\Theta}_{i}, \widehat{l}_{i}\right),\left(\bar{\Theta}_{i+1}, \bar{l}_{i+1}\right),\left(\Theta_{i+2}, l_{i+2}\right),\left(\Theta_{i+3}, l_{i+3}\right), \ldots$ is a $C^{\prime}(\lambda)-$ small cancellation labelling. Let $\widehat{\Theta}_{i+1}$ be the $\mathbb{Z}_{2}$-homology cover of $\bar{\Theta}_{i+1}$. Then the following properties are satisfied:

$$
\begin{aligned}
\left(A_{i+1}\right) & \left(\widehat{\Theta}_{1}, \widehat{l}_{1}\right), \ldots,\left(\widehat{\Theta}_{i}, \widehat{l}_{i}\right),\left(\widehat{\Theta}_{i+1}, \widehat{l}_{i+1}\right),\left(\Theta_{i+2}, l_{i+2}\right),\left(\Theta_{i+3}, l_{i+3}\right), \ldots \text { is } \\
& \text { a } C^{\prime}(\lambda) \text {-small cancellation labelling; } \\
\left(B_{i+1}\right) & \left(\widehat{\Theta}_{1}, \widehat{l}_{1}\right), \ldots,\left(\widehat{\Theta}_{i}, \widehat{l}_{i}\right),\left(\widehat{\Theta}_{i+1}, \widehat{l}_{i+1}\right) \text { admits a proper } \mathbb{Z}_{2}-\text { walling, }
\end{aligned}
$$

where $q_{i}: G_{i} \rightarrow G_{i+1}:=\left\langle S \mid\left(\widehat{\Theta}_{1}, \widehat{l}_{1}\right), \ldots,\left(\widehat{\Theta}_{i+1}, \widehat{l}_{i+1}\right)\right\rangle$ is the quotient map. Observe that we have $\operatorname{ker}\left(q_{i}\right)<K_{i}$.

The group $G_{i+1}=\left\langle S \mid \widehat{\Theta}_{1}, \widehat{\Theta}_{2}, \ldots, \widehat{\Theta}_{i+1}\right\rangle$ is hyperbolic and, by Lemma 2.3, acts geometrically on a CAT $(0)$ cubical complex. Hence, by results of Wise [Wis17] and Agol [Ago13], it is residually finite. Therefore, we find a map $\varphi_{i+1}: G_{i+1} \rightarrow F_{i+1}$ into a finite group $F_{i+1}$ such that $\varphi_{i+1}(g) \neq 1$ for all $g \in B_{i+1}\left(1, \operatorname{Cay}\left(G_{i+1}, S\right)\right.$ ). Hence, by $\left(C_{i}\right)$, we have

$$
\left(C_{i+1}\right) \varphi_{j}(g) \neq 1 \text {, for every } j \leqslant i+1 \text { and every } g \in B_{j}\left(1, \operatorname{Cay}\left(G_{j}, S\right)\right) \backslash\{1\} .
$$

For $j \leqslant i$ we define $\varphi_{i+1}^{j}: G_{i+1} \rightarrow F_{j}$ as $\varphi_{i+1}^{j}\left(q_{j}^{i+1}(g)\right)=\varphi_{j}(g)$. This is a well defined homomorphism: If $q_{j}^{i+1}(g)=q_{j}^{i+1}\left(g^{\prime}\right)$ then $q_{j}^{i}\left(g g^{\prime-1}\right) \in \operatorname{ker}\left(q_{i}\right)$, and hence

$$
\begin{aligned}
\varphi_{i+1}^{j}\left(q_{j}^{i+1}(g)\right)\left(\varphi_{i+1}^{j}\left(q_{j}^{i+1}\left(g^{\prime}\right)\right)\right)^{-1} & =\varphi_{j}(g) \varphi_{j}\left(g^{\prime}\right)^{-1}= \\
& =\varphi_{j}\left(g g^{\prime-1}\right)=\varphi_{i}^{j}\left(q_{j}^{i}\left(g g^{\prime-1}\right)\right)=1,
\end{aligned}
$$

by $\operatorname{ker}\left(q_{i}\right)<K_{i}$. By $\left(D_{i}\right)$ and the definition of $\varphi_{i+1}^{j}$ we have:

$\left(D_{i+1}\right) \varphi_{l}^{j} \circ q_{k}^{l}=\varphi_{k}^{j}$, for all $j \leqslant k \leqslant l \leqslant i+1$ (that is, the part of the above diagram with all indexes at most $i+1$ is commutative).

This finishes the inductive step.

3.3 Proof of Main Theorem. The presentation $\left\langle S \mid \widehat{\Theta}_{1}, \widehat{\Theta}_{2}, \ldots\right\rangle$ is a graphical $C^{\prime}(\lambda)$-small cancellation presentation, by $(A)$. Thus, the Cayley graph $\operatorname{Cay}(G, S)$ contains isometrically embedded copies of all the graphs $\widehat{\Theta}_{i}$, by Lemma 2.1. That is, Cay $(G, S)$ contains a sequence of $D$-regular graphs of growing girth, and hence $G$ is non-exact, by [Wil11].

We show now that $G$ is residually finite. Take a non trivial element $g \in G$. Let $i$ be such an integer that $g \in B_{i}(1, \operatorname{Cay}(G, S))$. Then there exists $g^{\prime} \in G_{i}$ such that $q_{i}^{\infty}\left(g^{\prime}\right)=g$, and $g^{\prime} \in B_{i}\left(1, \operatorname{Cay}\left(G_{i}, S\right)\right)$. For the homomorphism $\varphi_{\infty}^{i}: G \rightarrow F_{i}$ into the finite group $F_{i}$ we have $\varphi_{\infty}^{i}(g)=\varphi_{\infty}^{i} \circ q_{i}^{\infty}\left(g^{\prime}\right)=\varphi_{i}\left(g^{\prime}\right) \neq 1$, by $(D)$ and $(C)$. This shows that $G$ is residually finite. 


\section{Acknowledgements}

I thank Ana Khukhro and Kang Li for suggesting the question. I am grateful to them, and to Thibault Pillon for useful remarks. I thank Damian Sawicki for pointing out mistakes in the earlier version of the manuscript. The author was partially supported by (Polish) Narodowe Centrum Nauki, grant no. UMO-2015/18/M/ST1/00050. The paper was written while visiting McGill University. The author would like to thank the Department of Mathematics and Statistics of McGill University for its hospitality during that stay.

Open Access This article is distributed under the terms of the Creative Commons Attribution 4.0 International License (http://creativecommons.org/licenses/by/4.0/), which permits unrestricted use, distribution, and reproduction in any medium, provided you give appropriate credit to the original author(s) and the source, provide a link to the Creative Commons license, and indicate if changes were made.

\section{References}

[Ago13] Ian Agol. The virtual Haken conjecture. Doc. Math. 18(2013), 1045-1087. With an appendix by Agol, Daniel Groves, and Jason Manning. MR3104553

[BO08] Nathanial P. Brown and Narutaka Ozawa. $C^{*}$-algebras and finitedimensional approximations, Graduate Studies in Mathematics, vol. 88. American Mathematical Society, Providence, RI, (2008). MR2391387

[Gro03] Misha Gromov. Random walk in random groups. Geom. Funct. Anal., (1)13 (2003), 73-146. MR1978492

[HW14] G. C. Hruska and Daniel T. Wise. Finiteness properties of cubulated groups. Compos. Math., (3)150 (2014), 453-506. MR3187627

[Osa14] Damian Osajda. Small cancellation labellings of some infinite graphs and applications, arXiv preprint arXiv:1406.5015 (2014)

[Pri89] Stephen J. Pride. Some problems in combinatorial group theory, GroupsKorea 1988 (Pusan, 1988), (1989), pp. 146-155. MR1032822

[Roe03] John RoE. Lectures on coarse geometry. University Lecture Series, vol. 31, American Mathematical Society, Providence, RI (2003). MR2007488

[Wil11] Rufus Willett. Property A and graphs with large girth. J. Topol. Anal., (3)3 (2011), 377-384. MR2831267

[Wis17] DANiEL T. WiSE. The structure of groups with quasiconvex hierarchy. Annals of Mathematics Studies, Princeton University Press, Princeton, NJ (2017) to appear

Damian Osajda, Instytut Matematyczny, Uniwersytet Wrocławski, pl. Grunwaldzki 2/4, 50-384 Wrocław, Poland dosaj@math.uni.wroc.pl

Institute of Mathematics, Polish Academy of Sciences, Śniadeckich 8, 00-656 Warsaw, Poland

Department of Mathematics and Statistics, McGill University, Montreal, QC H3A 0B9, Canada 
Received: March 24, 2017

Revised: October 4, 2017

Accepted: November 13, 2017 